\title{
Aging-associated changes of movement-related functional connectivity in the human brain
}

\author{
Nils Rosjat ${ }^{\mathrm{a}, \mathrm{b}}$, Liqing Liu ${ }^{\mathrm{a}, \mathrm{b}}$, Bin A. Wang ${ }^{\mathrm{a}}$, Svitlana Popovych ${ }^{\mathrm{a}, \mathrm{b}}$, Tibor Tóth ${ }^{\mathrm{b}}$, Shivakumar \\ Viswanathan $^{\mathrm{a}, \mathrm{c}}$, Christian Grefkes ${ }^{\mathrm{a}, \mathrm{c}}$, Gereon R. Fink ${ }^{\mathrm{a}, \mathrm{c}}$, Silvia Daun ${ }^{\mathrm{a}, \mathrm{b}}$ \\ ${ }^{a}$ Cognitive Neuroscience, Institute of Neuroscience and Medicine (INM-3), \\ Research Centre Jülich, D-52425 Jülich \\ ${ }^{b}$ Heisenberg Research Group of Computational Neuroscience - Modeling Neural Network Function, Institute \\ of Zoology, University of Cologne, D-50674 Köln \\ 'Department of Neurology, University Hospital Cologne, D-50937 Köln
}

\section{Correspondence to:}

\section{Prof. Dr. Silvia Daun}

Cognitive Neuroscience,

Institute of Neuroscience and Medicine (INM-3),

Research Centre Jülich, 52425 Jülich, Germany

Tel: +49-2461-61 8638

Fax: +49-2461-61 1518

Email: s.daun@fz-juelich.de 


\begin{abstract}
Motor performance declines with normal aging. Previous neuroimaging work revealed agingrelated general increases in neural activity, especially in the prefrontal and pre-motor areas, associated with a loss of hemispheric lateralization. However, the functional mechanisms underlying these changes and their relation to aging-associated motor decline to date remain elusive.
\end{abstract}

To further elucidate the neural processes underlying aging-related motor decline, we recorded EEG from younger and older subjects while they performed a finger-tapping task. As a measure of synchronization between motor areas, we computed the inter-regional phase-locking value which reflects functional connectivity between distinct neural populations.

Behavioral data revealed increased movement times in older subjects. EEG data showed that phase locking in the $\delta-\theta$ frequencies is a general, age-independent phenomenon underlying the execution of simple finger movements. In stark contrast, the extent of synchronization between motor areas significantly differed dependent upon age of subjects: multiple additional intra- and inter-hemispheric connections were observed in older subjects. Our data shed light upon the results of previous neuroimaging studies showing aging-related increases in neural activation. In particular, data suggest that the observed aging-dependent substantial intra- and interhemispheric reorganization of connectivity between the corresponding motor areas underlies the previously reported loss of lateralization in older subjects. The changes observed are likely to represent compensatory mechanisms aiming at preserved task performance in older subjects.

Keywords: phase-locking value, transient synchrony, electroencephalogram, loss of lateralization, HAROLD, voluntary finger-tapping 


\section{Introduction}

Aging leads to increased difficulties in the coordination of movements, reflected by, e.g., an increased variability in movement execution (e.g., Wu and Hallett, 2005; Seidler et al., 2010). The wealth of data showing that human motor performance is affected by normal aging is contrasted by the dearth of data providing information about the neural processes underlying these changes.

Several fMRI studies suggest that the neural activity associated with self-initiated movements changes during normal aging. An enhanced general activation, especially in the pre-frontal and pre-motor areas, has been reported (Sailer et al., 2000; Heuninckx et al., 2005; Ward et al., 2008), often referred to as the PASA (posterior-anterior shift in aging) phenomenon (Dennis and Cabeza, 2008). Furthermore, consistent with the HAROLD (Hemispheric Asymmetry Reduction in Older Adults) model, which proposes that reduced hemispheric asymmetry reflects a general aging-associated mechanism rather than a task-specific phenomenon (for a summary see Cabeza, 2002), reduced hemispheric asymmetry during unilateral movements (Sailer et al., 2000; Rowe et al., 2006; Langan et al., 2010) has been reported. The less lateralized activity patterns during cognitive or motor performance in older relative to younger adults are assumed to reflect either compensation, i.e. the recruitment of additional supporting brain regions to offset the effects of aging-related cognitive (or motor) decline (e.g. Cabeza et al., 1997; Nolde et al., 1998; Reuter-Lorenz et al., 2000) or, alternatively, an increased difficulty in recruiting specialized neural mechanisms (Mitrushina and Satz, 1991; Babcock et al., 1997; Cabeza, 2002).

To date, the neural mechanisms underlying these reported aging-related changes in neural activation remain elusive. They may result, for instance, from changes in inter-regional neural synchronization, the latter constituting a fundamental mechanism underlying motor and 
cognitive tasks (e.g. Fell et al., 2004; Popovych et al., 2016). Neural synchronization refers to the precise and coordinated timing of the activity of distinct neural populations (for a review, see Palva and Palva, 2012), which is fundamental for motor and cognitive function (e.g. Uhlhaas et al., 2010). Studies using EEG/MEG/single-unit/ECoG data across a variety of different motor tasks have found that oscillations of remote neural populations exhibit short periods of synchronized activity (for review see Singer, 1999; Singer, 2004; Uhlhaas et al., 2010, van Wijk 2012). The communication-through-coherence hypothesis posits that this transient synchronization reflects increased functional connectivity between distinct neural populations expressed by coherently coordinated firing patterns (Fries, 2005, 2015). This synchronization is believed to underlie the communication and coordinated representation of information between remote neural populations (Baker et al., 1997, 2001; Uhlhaas et al., 2010).

Here, we investigated whether and to what extent the age-related changes in neural activation, especially to the degree of hemispheric symmetry, are linked to changes in transient synchronization of neural oscillations between brain regions, as indexed by their phase relationship.

Phase locking of neural activity is a key process of neural synchronization. A general statistic to quantify phase locking in networks is the phase locking value (PLV) (Lachaux et al., 1999). The PLV is a measure of variability in the phase relationship of neural activity at two distinct locations. A large PLV implies low variability (nearly constant phase relation) and hence strong connectivity between two distant neural populations. In contrast, a low PLV is a sign of weak connectivity between two distinct neural populations. Task-related changes in the PLV relative to a baseline value (henceforth, rPLV) provide a means to uncover event-related 
changes in network connectivity relative to a stationary phase-locking reference (i.e., baseline).

To this end, here we analyzed EEG data recorded from younger and older participants, while they performed a simple finger-tapping task. We specifically asked whether the functional networks, defined using the relative phase locking values (rPLV), systematically vary between younger and older age groups during movement preparation, execution, and outcome evaluation.

\section{Materials and Methods}

Participants. We used EEG data of twenty-one younger healthy individuals (10F/11M, age: 22-35 years) as well as a group of thirty-one older healthy subjects (15F/16M, age: 60-78 years) from two earlier studies (Popovych et al., 2016 and Liu et al., 2017, respectively). In both groups, all participants were right-handed according to the Edinburgh Handedness Inventory (Oldfield, 1971), had normal color vision, and no history of psychiatric or neurological disease (as assessed by the Trail making test (Spreen and Strauss, 1998), the Mini-mental-status-test (Folstein et al., 1975), the Clock-drawing test (Agrell and Dehlin, 1998), and the Beck depression inventory (Beck et al., 1961). All participants gave their written informed consent prior to the study. Both studies were approved by the local ethics committee of the Faculty of Medicine at the University of Cologne.

Experimental design. We recorded EEG data of both groups of subjects while they performed a simple finger-tapping task. The task consisted of a Kornhuber-Deecke-type self-initiated tapping condition (Kornhuber and Deecke, 1965) where the subjects were asked to voluntarily press a button with their left or right index finger every 4-8s. They were requested to perform the movements in random order, i.e., without using a systematic pattern, with a roughly equal 
number of left and right finger movements. The subjects were asked to avoid conscious timeestimation and trial-balancing in order not to evoke brain activity unrelated to the motor task itself. Subjects also separately performed a visually-cued task, where a left or right button press was executed in response to a visual stimulus, and a vision-only task, which had the same stimuli as in the visually-cued task but where no actions were required (for full details see Popovych et al., 2016, Liu et al., 2017, Wang et al., 2017). The whole experiment lasted about $70 \mathrm{~min}$.

Our analysis was restricted to data acquired from the self-initiated condition, as this condition was uncontaminated by the processing of visual stimuli. Each participant performed between 80 and 90 trials with each hand in this condition.

EEG recording and preprocessing. During the experiments, we continuously recorded EEG data from 64 active Ag/AgCl electrodes (Brain Products GmbH, Munich, Germany), which were placed according to the international 10-20 system. Following the generally accepted practice, the reference electrode was placed at the left earlobe. Bipolar horizontal and left vertical electro-oculograms (EOG) were recorded using three of the 64 scalp electrodes (FT9, FT10 and TP10 in the 10-20 system). These were placed at the bilateral outer canthi and under the left eye to monitor eye movements and blinks. Before the experiment, we ensured that the impedance of the electrodes was below $15 \mathrm{k} \Omega$. The EEG signals were amplified, band-pass filtered in the frequency range $0.87-500 \mathrm{~Hz}$, and digitized at a sampling rate of 2.5 kHz. An acceleration sensor was attached to the tip of each index finger to detect the movement onset. We used these sensors instead of EMG electrodes, since the former are more sensitive to small movements and are not restricted to specific muscle groups. We also used the information from the acceleration signals to monitor the behavior of the subjects, for example, to exclude errors, such as mirror movements. We defined the onset of the finger 
movement as the instant of time at which the numerical time-derivative of the acceleration signal crossed a predefined threshold.

The acquired raw data were band-pass filtered from $0.5-48 \mathrm{~Hz}$ to remove slow voltage drifts from the data and down-sampled from $2.5 \mathrm{kHz}$ to $200 \mathrm{~Hz}$ to reduce the file size and thereby the computational time. Artifacts were removed by a semi-automatic procedure in EEGLAB by means of independent component analysis (ICA) (Makeig et al., 1996). Finally, the data were epoched to intervals centered around the movement onset. In the self-initiated tapping condition, the baseline, which is assumed to contain no movement-related EEG component, had to be located before the start of the Bereitschaftspotential (readiness potential; Shibasaki and Hallett, 2006). Thus, the epoch or trial was defined to be the interval $[-2500,+1500] \mathrm{ms}$ relative to movement onset with a baseline period of [-2500, -1500] ms.

For our analysis, we excluded trials containing EEG artifacts as well as trials that were preceded by an interval of less than $4 \mathrm{~s}$ from the previous button-press. Trials containing movements during the baseline period, as detected using the accelerometer, were also excluded. After excluding trials based on these criteria, we only included data from participants who had at least 30 correct trials per hand remaining. Based on these quality considerations, data from three younger subjects and seven older subjects were excluded from further analysis. Thus, EEG data from 18 younger and 24 older subjects were used for further processing.

Spatial filtering. A crucial pre-processing step was spatial filtering. A major challenge in estimating network relationships with EEG is the phenomenon of volume conduction, whereby signals from a particular neural generator can influence voltages at multiple distinct electrodes on the scalp due to conduction leading to spurious phase-locking between these 
electrodes. Spatial filtering using surface Laplacians has been shown to substantially reduce this problem, thereby enhancing spatial resolution and enabling analyses of electrodes close to the region of interest (Lachaux et al., 1999, Tenke et al., 2012). Therefore, our preprocessed data were re-referenced to small Laplacian reference in order to improve the spatial resolution of the signals and thus enhance their suitability for the subsequent connectivity analysis (Hjorth, 1975).

Phase-locking analysis. Following preprocessing, we transformed the epoched data to the time-frequency domain using Morlet wavelets (Lachaux et al., 1999) in a range of 2-48 Hz with a step size of $1 \mathrm{~Hz}$ (5 cycles). This time-frequency decomposition was performed using the Statistical Parametric Mapping toolbox (SPM12, Wellcome Trust Centre for Neuroimaging, London, UK) implemented in MatLab R2011b (The MathWorks Inc., Massachusetts, USA). Subsequently, we analyzed the phase information gained from the time-frequency decomposition using customized MATLAB scripts.

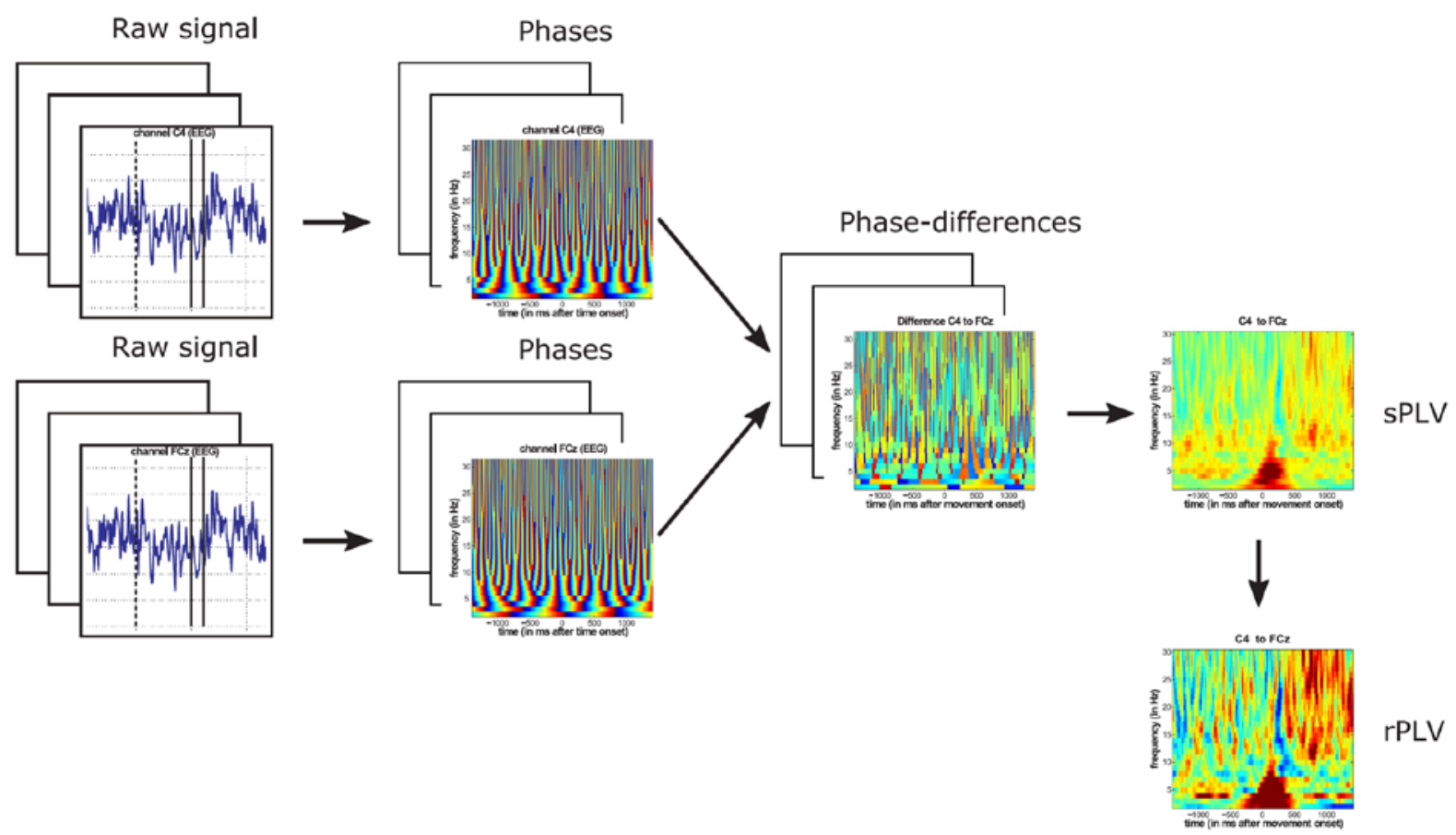

Figure 1: Schematic illustration of the procedure to calculate rPLV. See text for details. 
The complex Morlet wavelet transformation was used to compute the time evolution of the amplitude A(f, t) and phase $\phi(f, t)$ for each frequency separately. In order to quantify synchronization between the activities of the different brain regions, we used the singlefrequency phase-locking value (sPLV; adapting the phase-locking value defined in Lachaux et al., 1999, Fig. 1). For a pair of channels $m$ and $n$, sPLV is defined as:

$$
s P L V_{m, n}(f, t)=\frac{1}{N}\left|\sum_{k=1}^{N} \exp \left(i\left(\varphi_{m_{k}}(f, t)-\varphi_{n_{k}}(f, t)\right)\right)\right|
$$

Here $\varphi_{m_{k}}$ denotes the phase of the EEG at channel $m$ in the $k$-th trial. $N$ is the total number of trials, and $i$ is the imaginary unit. sPLV can take values in the interval $[0,1]$. While sPLV=0 represents a random distribution of phase differences over all trials without any coherence, sPLV=1 occurs only in the case of perfect inter-trial phase locking of the phase differences between the EEG signals at the two channels $m$ and $n$ over all trials.

Since we were interested in the synchronization effects during movement preparation and movement execution, and not in persistent phase synchronization, we normalized the sPLV of EEG at each pair of channels with respect to its baseline value and calculated its relative change over the whole epoch. We refer to these normalized sPLVs as 'relative phase-locking values’ (rPLV):

$$
r P L V_{m, n}(f, t)=\frac{s P L V_{m, n}(f, t)-\overline{s P L V_{m, n}(f)}}{\overline{s P L V_{m, n}(f)}}
$$


Here, $\overline{s P L V_{m, n}(f)}$ denotes sPLV of the baseline for each frequency, i.e., the mean sPLV at frequency $\mathrm{f}$ in the baseline interval. The baseline interval used to compute $\overline{s P L V_{m, n}(f)}$ was from [-2300, -1500] ms after excluding the first 200ms of the original [-2500,-1500] ms baseline as it contained edge artifacts of the Morlet transformation.

To analyze inter-regional synchronization of the oscillations recorded over different brain regions, we considered four major frequency bands: $\delta(2-3 \mathrm{~Hz}), \theta(4-7 \mathrm{~Hz}), \alpha(8-12 \mathrm{~Hz})$, and $\beta$ (13-30 Hz). A widely used procedure to evaluate phase locking in these frequency bands is to filter the signal to the frequency band of interest and calculate a single phase-locking value for the whole frequency band. Here, we instead calculated the rPLV for each single frequency separately with a resolution of $1 \mathrm{~Hz}$ before averaging these values over the frequencies of the frequency band. This was done to maximize the contribution of each individual frequency to the observed phase locking. Our primary focus was the average rPLV over the $\delta$ - $\theta$ frequency band $(2-7 \mathrm{~Hz})$ where we previously found evidence of considerably local phase locking (Popovych et al., 2016, Liu et al. 2017).

Network of interest. We chose to perform our connectivity analysis on the level of electrodes rather than on reconstructed sources in order to limit model-specific assumptions about sources of the observed oscillations. As our focus was on the dynamics of the motor network, our analysis was restricted to network relationships among ten electrodes clustered bilaterally over the premotor/sensorimotor cortex, namely, frontal F3/F4, fronto-central FC3/FCz/FC4, central C3/Cz/C4, centro-parietal CP3/CP4.

In our previous studies, the spatial distribution of local phase-locking in the $\delta-\theta$ frequency band $(2-7 \mathrm{~Hz})$ for unilateral movements was consistent with the lateralization of the motor system, namely, being higher at electrodes contralateral to the moved hand than at ipsilateral 
electrodes (Popovych et al., 2016; Liu et al., 2017). Based on this observation, the rPLV values of electrode pairs during left and right finger movements were collapsed together based on whether the electrodes were contralateral or ipsilateral to the moved hand. For convenience, electrodes over the right hemisphere were assumed to be contralateral to the moved hand while the left hemisphere was assumed to be ipsilateral.

The resulting network consisted of a total of 45 possible pairwise connections (Figure 2). Of the 45 possible connections, there were 14 intra-hemispheric ones per hemisphere (6 between non-medial electrodes and 8 between lateral and medial ones). One connection (FCz $-\mathrm{Cz}$ ) was strictly medial. The remaining 16 connections were strictly inter-hemispheric, i.e., between contralateral electrodes, 4 of them between mirror-symmetric electrodes.

Intra-hemispheric

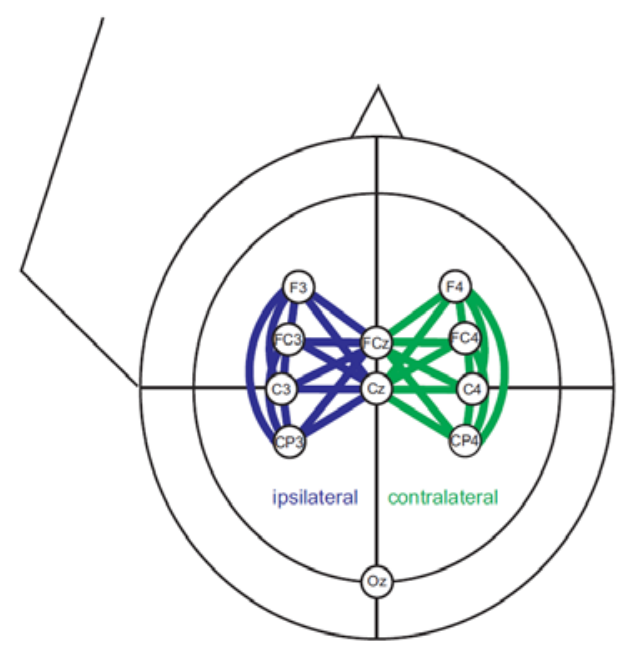

Inter-hemispheric

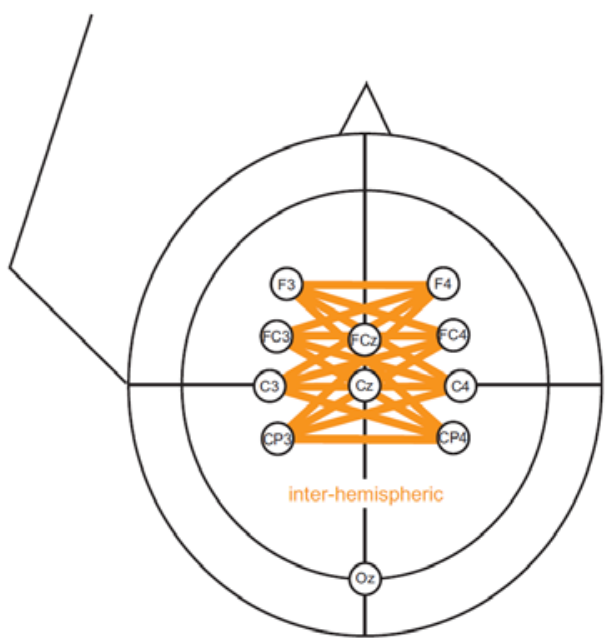

Figure 2: Summary of all possible connections in the network. 14 possible ipsilateral intrahemispheric connections (blue) and 14 possible contralateral intra-hemispheric connections (green) are shown on the left and 16 possible inter-hemispheric ones on the right (orange). 
We additionally included an occipital electrode $\mathrm{Oz}$ as a control electrode. As the task did not involve any task-relevant visual stimuli, the rPLV of connections from the electrodes of interest to $\mathrm{Oz}$ were used to assess the extent of spurious connectivity.

\section{Statistical analysis.}

To test whether the difference between the mean rPLV (averaged over frequency band) and the baseline was statistically significant, we compared the rPLV obtained for each pair of electrodes at each time point in the interval [-1500, 1500] ms with its baseline value using a pointwise t-test with a significance level of $\mathrm{p}<0.05$, corrected for multiple comparisons (false discovery rate, FDR, q = 0.05) (Benjamini and Hochberg, 1995) (Figure 3, middle column). The correction was performed with regard to the number of conditions, time points, age groups, and electrodes. The baseline was constructed by generating normally distributed random values that had the same mean value and standard deviation as the EEG at each time point of the baseline interval [-2300, -1500] ms.

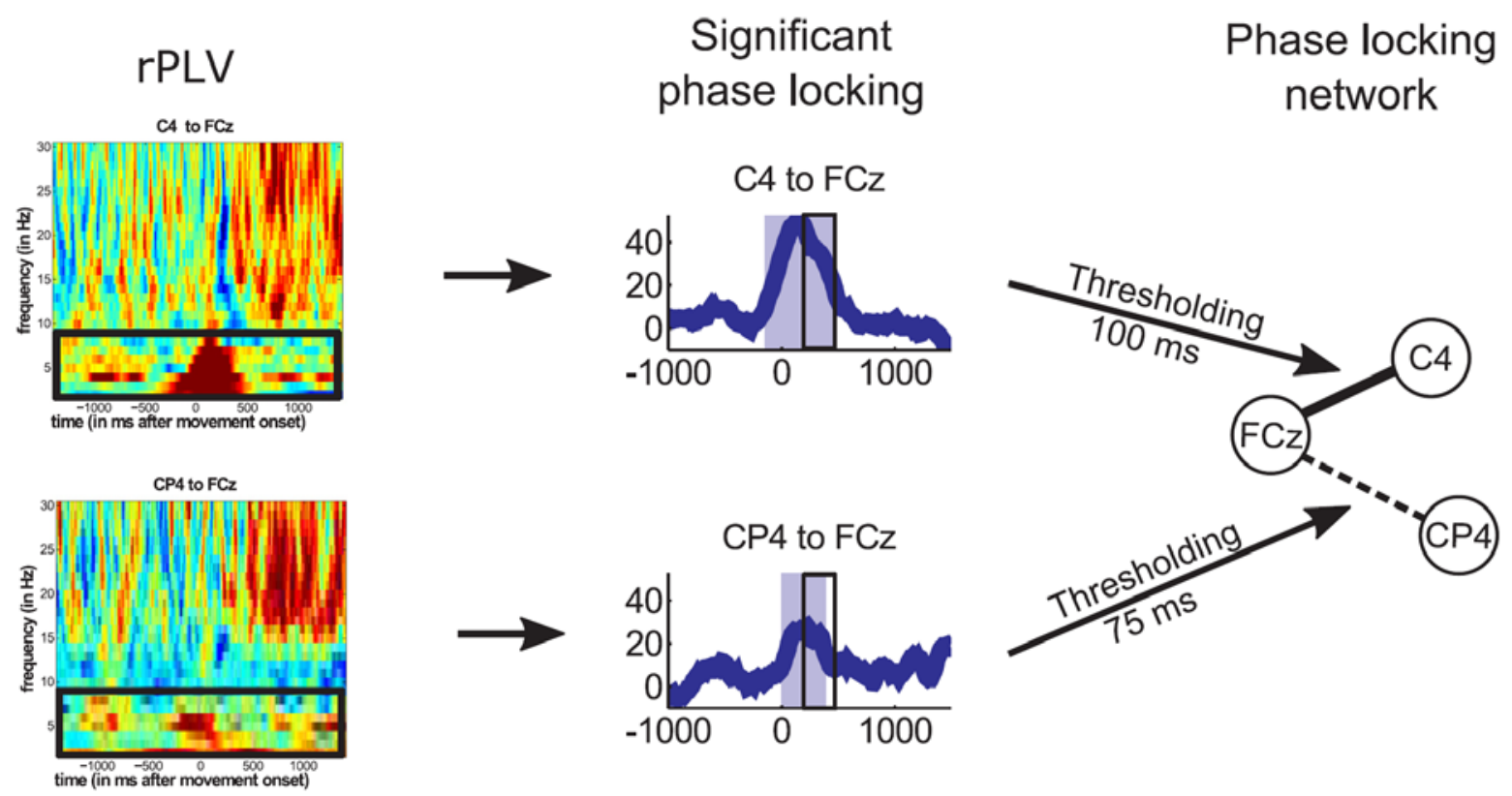

Figure 3: Construction of the corresponding phase locking networks from the rPLVs. See text for details. 
For the construction of the rPLV networks, we defined a 'connection', i.e., an edge of the network, between two given nodes to exist if rPLV between these two nodes was significant. Our analysis was restricted to the time interval $[-300,500] \mathrm{ms}$, which was divided up into a preparatory phase $[-300,0] \mathrm{ms}$, an execution phase of the movement $[0,200] \mathrm{ms}$, and a postmovement phase $[200,500]$ ms. Moreover, we split the whole interval $[-300,500]$ ms into bins of 100 ms length in order to assess the dynamics of the transient synchrony between the electrode pairs of interest. Two electrodes were regarded to be connected in such a subinterval if rPLV between them was significant over the whole duration $(100 \mathrm{~ms})$ of this interval (solid line), or at least for $75 \mathrm{~ms}$ in this time interval (dashed line in Figure 3, right column).

\section{Results}

Behavioral results.

We first tested whether motor performance differed between groups. To this end, we measured the movement time, i.e., the time from movement onset (determined using the accelerometer) to the actual button press. We tested the movement times of younger $(\mathrm{M}=81.9 \mathrm{~ms}$, Std $=8.6)$ vs. older $(\mathrm{M}=88.3 \mathrm{~ms}$, Std $=8.3)$ subjects for significant differences and found that the finger movements of older subjects were significantly (on average $6 \mathrm{~ms}$ ) slower than those of the younger ones $(\mathrm{t}(82)=-3.4307, \mathrm{p}=0.0004)$.

\section{Event-related increase in phase locking.}

Prior to any test of age-related differences, we first sought to evaluate whether the expected task-induced changes in phase-locking were statistically detectable at all over the electrode pairs in the motor network (Figure 2). This was indeed the case:

For both populations, the relative phase-locking value (rPLV) in the $\delta-\theta$ frequency band showed a significant increase over the baseline pre-, during, and post-movement ( $\mathrm{t}=0$ denotes 
movement onset) for many electrode pairs (increase in rPLV up to 50\%)(Figure 4). Significant phase locking occurred mainly between movement-related electrodes (see Figure 4, rPLV between ipsilateral, contralateral, and inter-hemispheric pairs of electrodes), in both younger and older subjects. Importantly, phase-locking between electrodes in the motor network and the occipital control electrode $\mathrm{Oz}$ was comparatively negligible (bottom panel, Figure 4). The electrode $\mathrm{Oz}$ was omitted from further analysis.
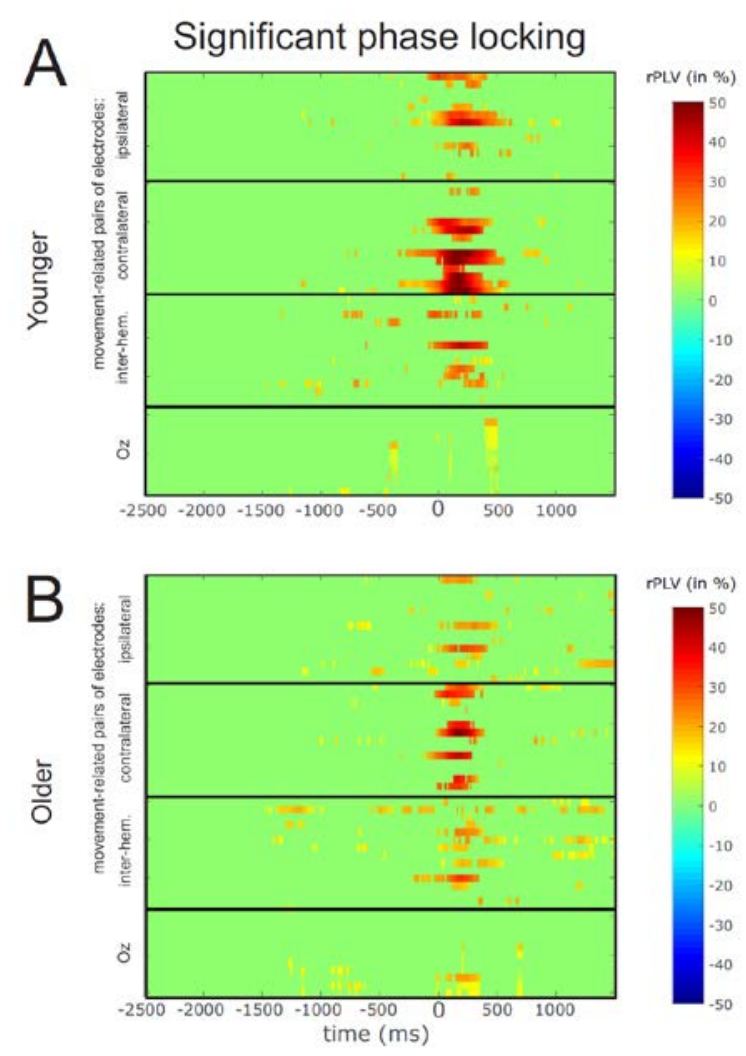

Figure 4: Colormap showing the time evolution of the rPLV for all electrode pairs of interest (cf. Methods, Figure 1). The upper part of each of the two subfigures displays the rPLV between movement-related electrodes (divided into ipsilateral, contralateral, and interhemispheric electrode pairs) and the bottom part of each subfigure the one between movement-related electrodes and $\mathrm{Oz}(\mathrm{Oz})$ in younger $(\mathrm{A})$ and older subjects $(\mathrm{B})$ in the $\delta-\theta$ frequency band, averaged over 2-7 Hz. Only significant rPLV values are shown with their 
respective colors ( $\mathrm{p}<0.05$, FDR corrected, cf. Methods). Non-significant ones are displayed in green ( $0 \%$ change). $\mathrm{t}=0$ denotes movement onset.

Since our analysis revealed that inter-regional phase locking in the $\delta-\theta$ frequency band was indeed movement-related, occurring in both younger and older subjects, we next turned to how these phase dynamics differ between these populations.

Differences in phase locking due to age.

To investigate differences in transient synchronization between younger and older subjects, we first analyzed the time evolution of synchronization in both age groups separately. The rPLV was used to construct network snapshots of the dynamic connectivity process during the different stages of the action (i.e., preparation, execution, and post-movement) defined by each of eight $100 \mathrm{~ms}$ subintervals from $-300 \mathrm{~ms}$ before movement onset to $+500 \mathrm{~ms}$ after movement onset (Figure 5). If the rPLV was statistically significant between two nodes of the network, we defined this relationship to be a “connection” in the network (see Methods). 


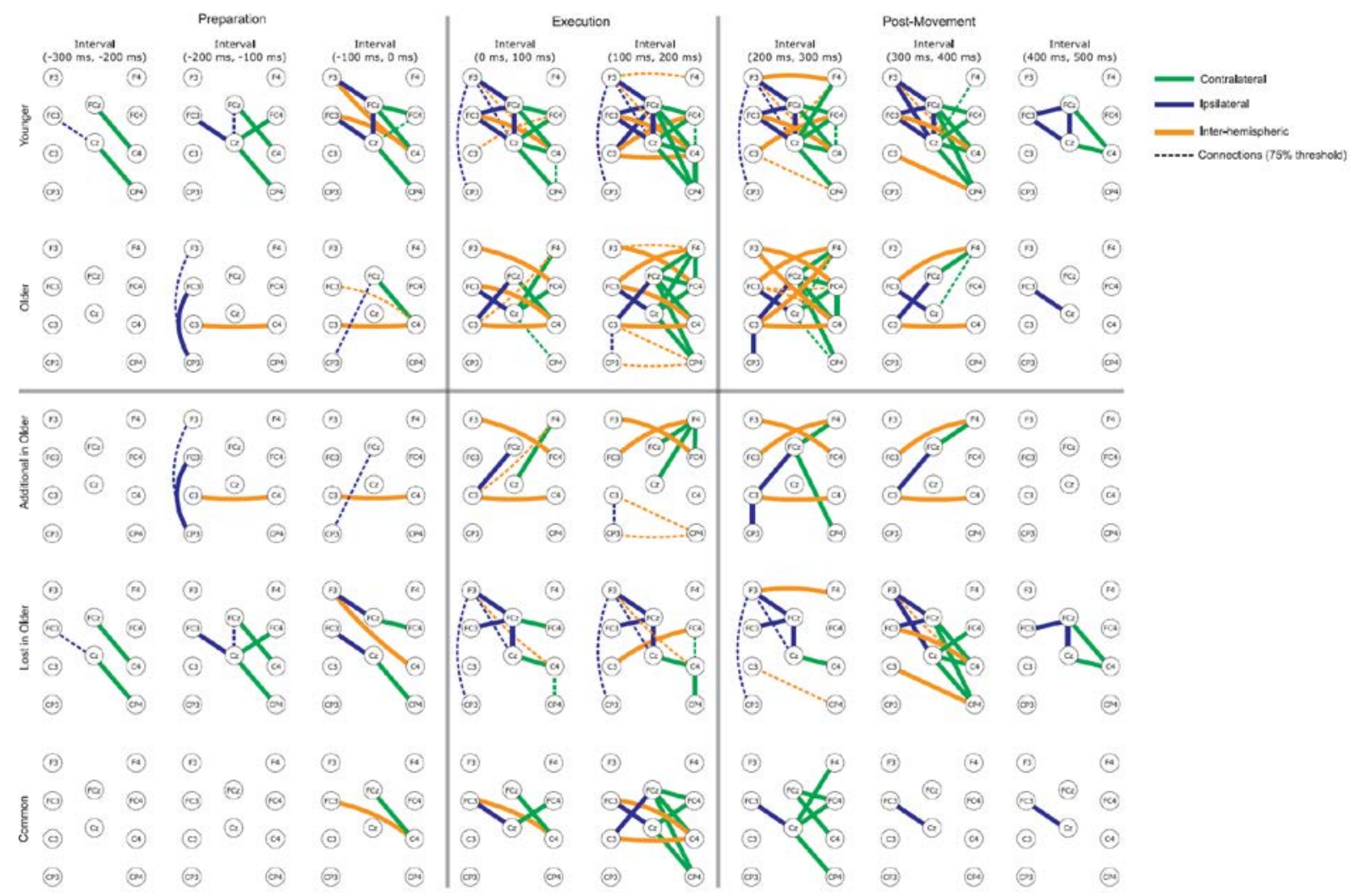

Figure 5: Time evolution of rPLV networks. The networks are computed from EEG signals at the 10 electrodes of interest in the interval [-300,500] ms divided into 8 subintervals of 100 ms length. Significant inter-regional phase locking (rPLV) of EEG between electrode pairs for the whole 100 (or at least 75) ms is represented by an edge drawn in solid (or dashed) line. Results on the left and right index finger-tapping movements have been merged and are shown in the two upper rows for younger and older subjects, as indicated. Connections are classified as ipsilateral (blue), contralateral (green), and inter-hemispheric (orange). The 2nd and 3rd row depict the connectivity changes in the older subjects by displaying additional connections and lost connections, respectively. The last row shows the connections that are common to both age groups.

For each age-group, the networks defined by connections that survived continuously over the entire $100 \mathrm{~ms}$ of each subinterval (solid lines) as well as those that only survived for $75 \mathrm{~ms}$ of 
that subinterval (dashed lines) are shown in Figure 5, first row (younger participants) and second row (older participants). Moreover, we distinguished 3 types of inter-regional phase locking: inter-hemispheric (orange), ipsilateral (blue), and contralateral connections (green). Rather than a uniform loss or gain of connections, the network for older participants exhibited a complex relationship to that of the younger participants showing both a gain in connections (Figure 5, third row) while accompanied by a loss of other connections (Figure 5, fourth row). Only a small fraction of connections were shared across both groups over all subintervals (Figure 5, last row).

When lowering the threshold for connectivity (see Methods) in our analysis from $100 \mathrm{~ms}$ to 75ms, we observed that the resulting networks underwent only minor changes, i.e., a maximal increase by five edges per time-interval was observed (see dashed lines in Figure 5). Importantly, these minor changes had virtually no bearing on the network properties reported here. The network properties in question can thus be regarded as robust against small-tomoderate changes of the threshold.

We next tested whether the strength of phase locking differed between the two groups. Our test was restricted to connections that survived for at least one $100 \mathrm{~ms}$ interval in either age group, namely, all connections displayed in the first and second rows of Figure 5 (solid lines). Age-related modulations of the mean rPLV of the above connections over each $100 \mathrm{~ms}$ interval was assessed with a three-way ANOVA with factors Age Group \{Young, Old $\mathrm{x}$ Time \{8-subintervals x Connection \{23-pairs $\}$. The 3-way interaction between these factors was not statistically significant $[F(154,15242)=0.44, p>0.05]$. However, we found a significant interaction between age group and connection $(\mathrm{F}(22,15242)=11.75, \mathrm{p}<0.0001)$. The main effects of all factors were also statistically significant [age group: $(\mathrm{F}(1,15242)=64.52, \mathrm{p}<$ 0.0001); time: $\mathrm{F}(7,15242)=64.4, \mathrm{p}<0.0001$; connection: $(\mathrm{F}(22,15242)=15.5, \mathrm{p}<0.0001)]$. 
A posteriori t-tests on these mean rPLVs revealed that the EEG of younger subjects exhibited significantly higher phase locking in 30 pairs of electrodes in various time intervals than the EEG of older subjects (Figure 6 top row, solid lines) (all ts(82) $>1.7622$, all ps $<0.05$, FDR corrected $q=0.05)$. The EEG of older subjects showed an increased phase locking in only 7 pairs of electrodes (all ts $(82)<-1.7131$, all ps $<0.05$ FDR corrected $q=0.05$ ) relative to that of younger subjects (Figure 6 top panels, broken lines).

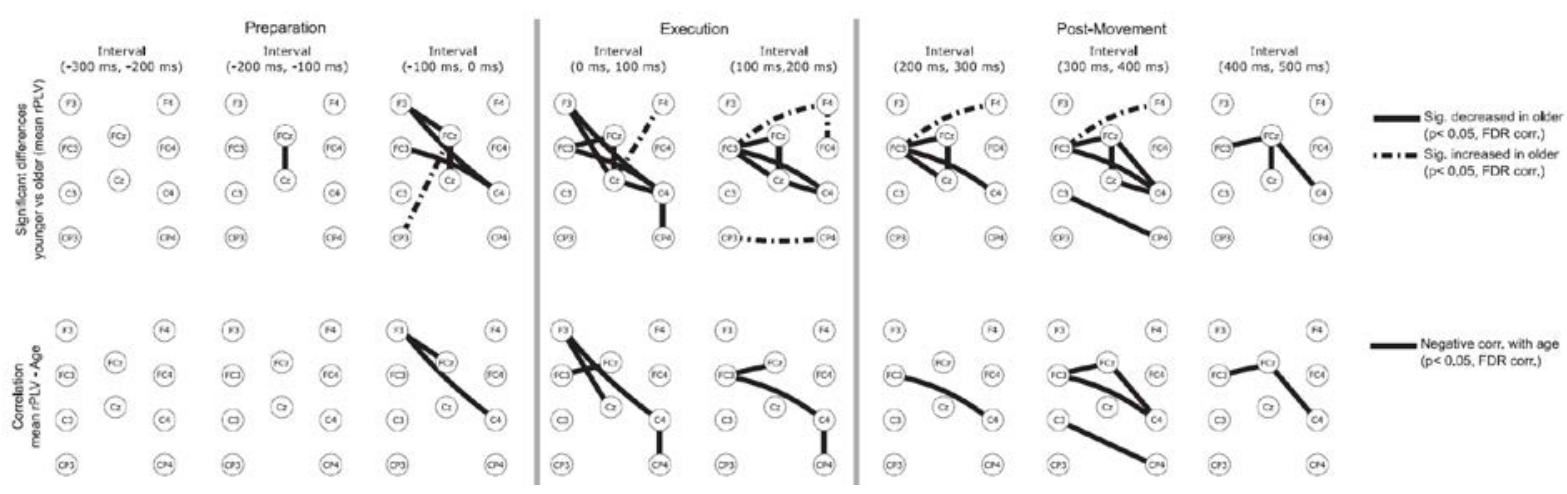

Figure 6: Results of the statistical analysis. Top row: connections for which rPLV was significantly different between age groups. Solid lines depict rPLVs that were significantly decreased, dashed lines the ones that were significantly increased in older subjects. Bottom row: Connections for which rPLVs correlated negatively with the age of the subjects.

\section{Correlation between age and rPLV.}

The strength of mean rPLV differed between the age groups for several connections as evident in Figure 6 (top panel). To further assess the age-dependence of mean rPLV strength at each of these connections, we pooled the two populations together and tested the correlation between the numerical age and mean rPLV (averaged in each of the $100 \mathrm{~ms}$ time intervals) for these connections. There was a significant negative correlation between age and inter-regional phase-locking (i.e., rPLV) for several electrode pairs, namely, F3 - FCz, F3 - C4, FC3 - FCz, 
FC3 - C4, C4 -CP4, C3 - CP4 and FCz - C4 (all $\rho s<-0.2467$, all ps $<0.05$, FDR corrected q $=0.05$ ) (Figure 6, bottom row). That is, the older the subjects, the smaller was the rPLV for those connections.

Since movement time was significantly higher for the older group relative to the younger group (see Behavioral results above), we also tested whether rPLV was correlated with movement time. However, this correlation was not statistically significant suggesting that the age-dependence of the rPLV did not directly arise due to movement differences.

\section{Betweenness centrality.}

Applying the search procedure for hub nodes in networks (i.e., nodes through which the most paths with the shortest length pass (Bullmore and Sporns, 2009)), which are usually the ones with the largest degree, to the networks in Figure 5 suggests that the brain region at both nodes $\mathrm{Cz}$ and $\mathrm{FCz}$ revealed hub-like properties during the preparatory, the executional, and the post-movement stages, both in younger and in older subjects.

\section{Network summary.}

To summarize the multiple network changes revealed by changes in phase-locking, we made some simplifying anatomical assumptions: We assumed that the channels C4 and CP4 represent activity of the contralateral sensorimotor cortex (cSM), C3 and CP3 that of the ipsilateral sensorimotor cortex (iSM). The frontal and fronto-central electrodes F4 and FC4 were assumed to represent activity from the contralateral prefrontal and pre-motor cortex (cPFC/cPM), and similarly, electrodes F3/FC3 represent ipsilateral iPFC/iPM. Finally, the central electrodes $\mathrm{Cz}$ and $\mathrm{FCz}$ were deemed to reflect activity related to the medial pre-frontal cortex and the supplementary motor area (mPFC/SMA). Based on these simplifying assumptions, Figure 7 summarizes the results obtained for the network of ten electrodes in 
Figure 5 in a compact form. As in Figure 5, edges between two brain regions denote significant, temporary inter-regional phase locking that existed in a given phase of the movement (preparation, execution, and post-movement). A more detailed view of the networks in the case of separately analyzed left and right hand tappings can be found in the supplementary material (see Fig. S1).

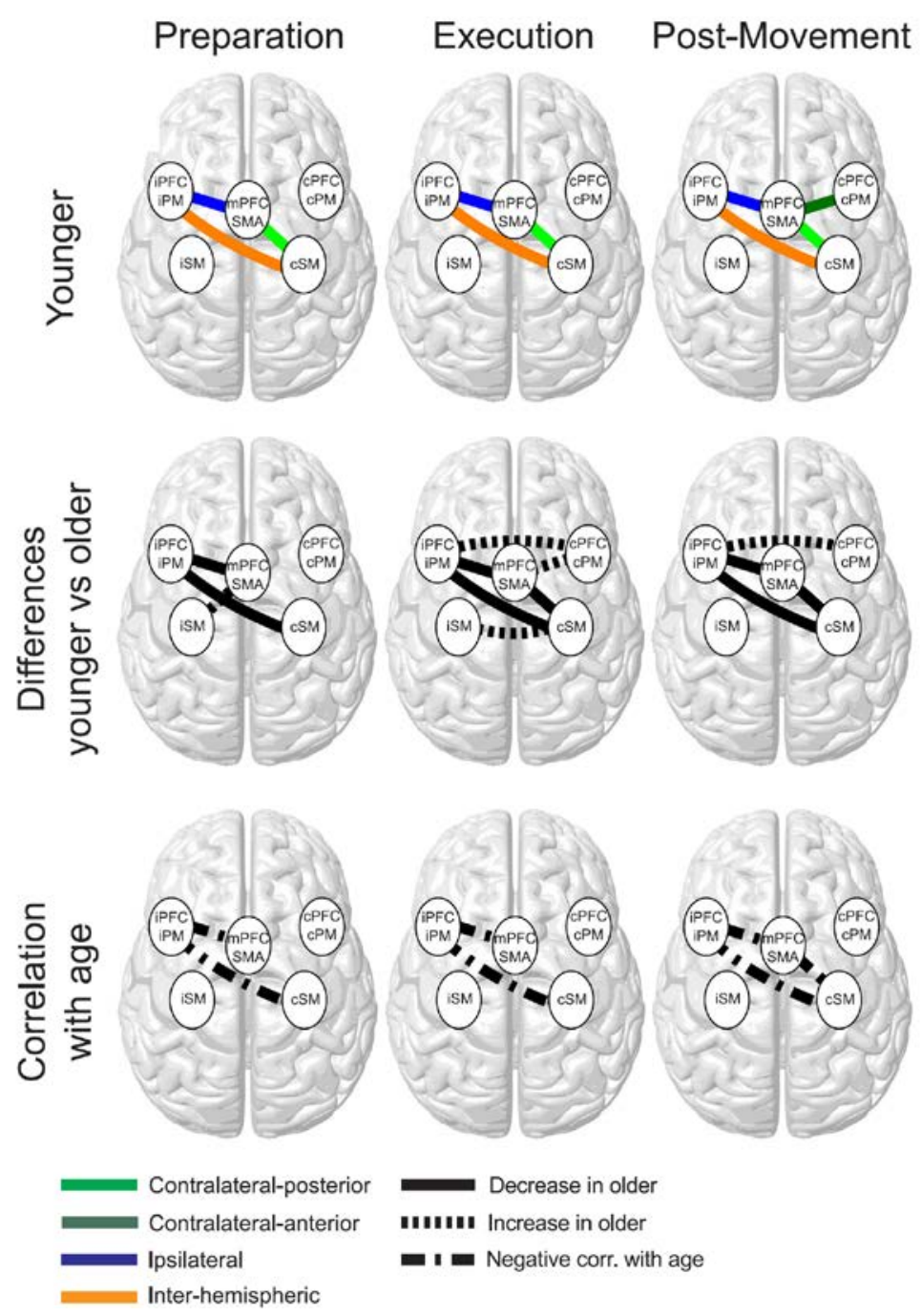

Figure 7: Summary of the results presented as a network of five virtual electrodes: younger subjects (first $=$ upper row); significant differences between connections (rPLVs) in younger and older subjects (second row); and connections that significantly correlated with age (third row). The networks in each row appeared during the preparation $[-300,0]$ ms, the execution phase of the movement $[0,200] \mathrm{ms}$, and in the post-movement phase [200,500] ms. 
Connections are labeled as follows: ipsilateral (blue), contralateral SM-mPFC/SMA (light green), contralateral PFC/PM-mPFC/SMA (dark green), and inter-hemispheric (orange). Significantly decreased connections (rPLVs) are shown as solid lines, significantly increased connections as dashed lines, and correlations of rPLVs with age as dash-dotted lines. The connections in the top row are drawn using the same color-codes as in Figure 4, with the additional distinction between connections from contralateral SM (cSM) to mPFC/SMA (light green) and those from contralateral PFC/PM (cPFC/cPM) to mPFC/SMA (dark green).

In this simplified form, we see that the motor network for younger subjects (top row, Figure 7) had an invariant sub-network consisting of the connections iPFC/iPM - mPFC/SMA (blue), mPFC/SMA - cSM (light green) and iPFC/iPM - cSM (orange), which persisted in all phases of the movement. Only in the post-movement phase, an additional edge between cPFC/cPM and mPFC/SMA (dark green) appeared in the network.

While some of the connections representing phase locking and being present in younger subjects were significantly reduced in older ones (Figure 7, second row, black solid lines), certain other connections emerged (Figure 7, second row, dashed lines). For instance, an additional connection between iSM and mPFC/SMA was established in the preparatory phase of the movement. During movement execution three connections showed an increased phase locking in older subjects: a contralateral edge connecting cPFC/cPM and mPFC/SMA, an inter-hemispheric edge connecting iSM and cSM, and an inter-hemispheric edge connecting $\mathrm{iPFC/iPM}$ and cPFC/cPM, the latter being also present in the post-movement phase.

In addition to the significantly decreased phase locking observed in older subjects for the connections that form the sub-network in younger subjects, we found significant correlations of the mean rPLV of these connections with the age of the subjects in all phases of the 
movement. That is, the older the subject the weaker was the phase locking between iPFC/iPM and mPFC/SMA and between iPFC/iPM and cSM in all movement phases. Furthermore, the phase locking between mPFC/SMA and cSM was negatively correlated with age in the postmovement phase.

In summary, we observed a rich variety of dynamic networks representing synchronization between different brain regions. The majority of the edges lasted over several phases of the movement and, importantly, there were characteristic effects of age.

\section{Discussion}

We carried out inter-regional phase-locking analyses on EEG data obtained from younger and older healthy subjects, while they performed a simple self-initiated finger-tapping task. We compared the time evolution of inter-regional phase locking between the participating brain areas during preparation, execution, and post-movement to assess periods of transient synchronization between the involved neural populations. Phase locking was assessed, since it constitutes a key process underlying the communication between neural populations (Moore et al., 2006; Schmorrow and Fidopiastis, 2011). According to the communication through coherence (CTC) hypothesis, synchronization reflects increased functional connectivity between neural populations expressed by coherently coordinated firing patterns (Fries, 2005, 2015).

To gain an overall picture of the functional connectivity during motor task performance, we calculated the rPLV, which measures the extent of instantaneous synchronization between two distinct brain regions. We thereby identified a network of areas associated with the motor task. The great advantage of the rPLV is that it is capable of extracting information from fast transient signals. Other methods, like dynamic causal modeling (DCM) or Granger causality, 
require statistically stationary signals. To start with, the nodes were assigned to electrodes of interest and the edges represented significant inter-regional rPLVs computed in the $\delta-\theta$ frequency band from the EEGs at these electrodes in all movement phases (Figure 4). We thus obtained dynamic networks that describe the temporal changes in functional connectivity in the different movement phases. Special nodes, so-called hubs, essentially nodes with largest degrees, were identified within these networks. During almost the whole length of the movement, $\mathrm{FCz}$ and $\mathrm{Cz}$ turned out to constitute such hub nodes, in both age groups. Moreover, one node occasionally took over this role from the other one. This hub-property emphasizes the functional significance of the central brain region, where the medial PFC and the SMA reside, in self-initiated motor actions.

Although a reconstruction of source activity would probably have provided more detailed information on the origin of the oscillations, we performed our connectivity analysis on the level of electrodes. We did so in order to avoid the ill-posed inverse problem, which is only an assumption of the source activity and could thus lead to flawed interpretations (Bradley et al., 2016; Grech et al., 2008; Wendel et al., 2009). We used the small Laplacian reference to substantially reduce the effect of volume conduction on our data (Lachaux et al., 1999). Though not an explicit source localization method, the small Laplacian reference renders the electrodes maximally sensitive to radial sources that directly lie beneath them (Rigoni et al., 2013; Steinmetz et al., 1989; Tenke et al., 2012). Another way of dealing with the problem of common sources could have been to compute measures that are not sensitive to 0-phase-lag interactions, e.g. weighted phase-lag index or imaginary PLV (Vinck et al., 2011; Nolte et al., 2004). Even so, our analysis revealed only a small number of neighboring electrodes that were connected (as opposed to all 45 possible connections cf. Figs. 1 and 3). Furthermore, neighboring connections that were present in younger subjects were absent in older ones. It is 
therefore unlikely that the reported connections would solely result from volume conduction. They are much more likely to arise from distinct brain sources.

At the second stage, we identified invariant sub-networks that existed in all or almost all phases of the movement. These sub-networks occurred in both age groups, albeit that they differed. In particular, in the group of younger subjects, the edges iPFC/iPM-mPFC/SMA, $\mathrm{mPFC} / \mathrm{SMA} \longrightarrow \mathrm{CSM}$, and $\mathrm{iPFC} / \mathrm{iPM}-\mathrm{CSM}$ were observed. This is in basic agreement with fMRI connectivity data in healthy younger participants who performed a left- or right-handed fist closure task. Here, a connectivity increase from SMA to cSM was observed (Grefkes et al., 2008; Pool et al., 2013). However, given the poor time resolution of fMRI, such studies could not identify the connectivity networks that were active during the different phases of a simple and rapid motor task (i.e., preparation, execution, and post-movement evaluation).

In older subjects, in contrast to younger ones, the strength of these rPLVs, i.e., the strength of the connections forming this strongly lateralized sub-network, was significantly reduced. Conversely, phase locking between iM1 and the central areas as well as between contralateral frontal and the central areas was significantly higher than in younger subjects. Additionally, older subjects showed a significant increase in inter-hemispheric phase locking, i.e., between $\mathrm{iSM}-\mathrm{CSM}$ and $\mathrm{iPFC} / \mathrm{iPM}-\mathrm{CPFC} / \mathrm{cPM}$. All this led to a decrease in hemispheric asymmetry (i.e., a loss of lateralization) in the motor and prefrontal cortex of older subjects. The data are compatible with a DCM analysis of fMRI data reported by Boudrias and colleagues (Boudrias et al., 2012), which revealed that older subjects display stronger connections onto iSM from cSM.

Further fMRI studies showed a strong general activation, especially in the PFC and the (pre) motor areas (Sailer et al., 2000; Heuninckx et al., 2005; Ward et al., 2008), as well as a loss of 
lateralization in older subjects (Sailer et al., 2000; Rowe et al., 2006; Langan et al. 2010). Based on our results, we hypothesize that the changes observed in neural activation result, at least in part, from changes in phase locking, i.e., from altered patterns of transient synchronization between the participating brain regions. Specifically, our data suggest that i) the diminished synchronization in the lateralized sub-network (iPFC/iPM - mPFC/SMA cM1), and ii) the increase in intra- and inter-hemispheric connectivity (i.e., iSM-mPFC/SMA, mPFC/SMA-cPFC/cPM, iSM-cSM and iPFC/iPM-cPFC/cPM), underlie the reported loss of lateralization in older subjects. Thus, there is not only an increase in overall activity, which is commonly interpreted as a loss of lateralization in the power spectral density (Sailer et al., 2000), but also a substantial reorganization in functional connectivity between the corresponding areas in both hemispheres.

In an earlier study (Popovych et al., 2016), we found an increase in local phase locking in the $\delta-\theta$ frequency band of EEG at the electrodes that lie above the motor areas of the cortex contralateral to the moving hand. We hypothesized that the preparation and execution of motor actions, irrespective of whether the movement was initiated internally or by an external cue, rely on local phase locking in the motor cortex prior to the actual execution, i.e., during preparation. In our follow-up study (Liu et al., 2016), we found that this local phase locking is age independent. The fact that local synchronization is age independent while the interregional one changes quite drastically with age is compatible with the 'network view' of the HAROLD model. There, it is assumed that an age-related increase in activation in a certain brain region is not independent of the effects of aging in other brain regions involved in a certain task, i.e., that it rather results from a global network change.

Summing up, we found that inter-regional phase locking in the $\delta-\theta$ frequency band between and within the frontal and motor regions underlies the execution of a finger tapping task. 
Since enhanced activity of, and also increased connectivity between, brain regions is often interpreted as functional compensation in older individuals (Reuter-Lorenz and Cappell, 2008; Grady, 2012), we suggest that the observed increase in the inter-hemispheric and cPFC/cPMmPFC/SMA and mPFC/SMA-iSM connectivity in older subjects constitutes a compensatory mechanism that aim at counteracting the loss of the strongly lateralized sub-network (iPFC/iPM-mPFC/SMA, mPFC/SMA-cSM, iPFC/iPM-cSM) in the different phases of the motor act.

Older subjects showed a significant increase (about $6 \mathrm{~ms}$ ) in movement time, i.e., the time from movement onset to the button press. One reason for increased movement times might be the reduced conduction velocity of nerve fibers in older subjects (Nishihara et al., 2013). Another reason could be, since we did not find any correlation between rPLV and movement time, that the brain of older subjects compensated for the loss in iPFC/iPM-mPFC/SMA-cSM connectivity by establishing additional intra- and inter-hemispheric connections without which subjects would have performed worse (i.e., when relying solely on the sub-network present in younger subjects only). Our results indicate that not a single connection but an assembly of additionally established connections is drawn upon in the attempt to counteract the naturally occurring decline of motor functions. Our results therefore support the compensation hypothesis of the HAROLD model, which proposes that age-related asymmetry reductions may help to counteract neurodegenerative decline. Finally, our results did not reveal evidence for the realization of the PASA phenomenon through phase locking in the $\delta-\theta$ frequency range.

\section{Role of the funding source}

This work was funded by the University of Cologne Emerging Groups Initiative (CONNECT group) within the framework of the Institutional Strategy of the University of Cologne and the 
German Excellence Initiative. SD gratefully acknowledges additional support from the German Research Foundation (GR3690/2-1, GR3690/4-1, DA1953/5-2). GRF gratefully acknowledges additional support by the Marga and Walter Boll foundation.

\section{Conflict of interest}

The authors declare no competing financial interests. 


\section{References}

Agrell B, Dehlin O (1998) The clock-drawing test. Age and Ageing 27:399-403.

Babcock RL, Laguna KD, Roesch SC (1997) A comparison of the factor structure of processing speed for younger and older adults: Testing the assumption of measurement equivalence across age groups. Psychology and Aging 12:268-276.

Baker SN, Olivier E, Lemon RN (1997) Coherent oscillations in monkey motor cortex and hand muscle EMG show task-dependent modulation. The Journal of physiology 501(1):225241.

Baker SN, Spinks R, Jackson A, Lemon RN (2001) Synchronization in monkey motor cortex during a precision grip task. I. Task-dependent modulation in single-unit synchrony. Journal of Neurophysiology 85(2):869-885.

Beck AT, Ward CH, Mendelson M, Mock J, Erbaugh J (1961) An inventory for measuring depression. Arch Gen Psychiatry 4:561-571.

Benjamini Y, Hochberg Y (1995) Controlling the false discovery rate: a practical and powerful approach to multiple testing. Journal of the Royal Statistical Society Series B (Methodological):289-300.

Boudrias MH, Gonçalves CS, Penny WD, Park CH, Rossiter HE, Talelli P, Ward NS (2012) Age-related changes in causal interactions between cortical motor regions during hand grip. Neuroimage 59(4):3398-3405. 
Bradley A, Yao J, Dewald J, Richter CP (2016) Evaluation of electroencephalography source localization algorithms with multiple cortical sources. PloS One 11(1):e0147266.

Bullmore E, Sporns O (2009) Complex brain networks: graph theoretical analysis of structural and functional systems. Nature Reviews Neuroscience 10(3):186-198.

Cabeza R (2002) Hemispheric Asymmetry Reduction in Older Adults: The HAROLD Model. Psychology and Aging 17(1):85-100.

Cabeza R, Grady CL, Nyberg L, McIntosh AR, Tulving E, Kapur S, Jennings JM, Houle S, Craik FIM (1997) Age-related differences in neural activity during memory encoding and retrieval: A positron emission tomography study. Journal of Neuroscience 17:391-400.

Dennis NA, Cabeza R (2008) Neuroimaging of healthy cognitive aging. In The Handbook of Aging and Cognition (Craik FIM, Salthouse TA, eds.), pp1-54. New York: Psychology Press, 3rd Edn.

Fell J, Dietl T, Grunwald T, Kurthen M, Klaver P, Trautner P, Schaller C, Elger CE, Fernandez G (2004) Neural bases of cognitive ERPs: more than phase reset. Journal of cognitive neuroscience 16(9):1595-1604.

Folstein MF, Folstein SE, McHugh PR (1975) “Mini-mental state”. A practical method for grading the cognitive state of patients for the clinician. Journal of Psychiatric Research 12(3):189-198. 
Fries P (2005) A mechanism for cognitive dynamics: neuronal communication through neuronal coherence. Trends in cognitive sciences 9(10):474-480.

Fries P (2015) Rhythms for cognition: communication through coherence. Neuron 88(1):220235.

Grady C (2012) The cognitive neuroscience of ageing. Nature Reviews Neuroscience 13(7):491-505.

Grech R, Cassar T, Muscat J, Camilleri KP, Fabri SG, Zervakis M, Xanthopoulos P, Sakkalis V, Vanrumste B (2008) Review on solving the inverse problem in EEG source analysis. Journal of neuroengineering and rehabilitation 5(1):25.

Grefkes C, Nowak DA, Eickhoff SB, Dafotakis M, Küst J, Karbe H, Fink GR (2008) Cortical connectivity after subcortical stroke assessed with functional magnetic resonance imaging. Annals of neurology 63(2):236-246.

Heuninckx S, Wenderoth N, Debaere F, Peeters R, Swinnen SP (2005) Neural basis of aging: the penetration of cognition into action control. Journal of Neuroscience 25(29):6787-6796.

Hjorth, B. (1975). An on-line transformation of EEG scalp potentials into orthogonal source derivations. Electroencephalography and clinical neurophysiology, 39(5), 526-530.

Kornhuber HH, Deecke L (1965) Hirnpotentialänderungen bei Willkürbewegungen und passiven Bewegungen des Menschen: Bereitschaftspotential und reafferente Potentiale. Pflügers Archiv European Journal of Physiology 284(1):1-17. 
Lachaux JP, Rodriguez E, Martinerie J, Varela FJ (1999) Measuring phase synchrony in brain signals. Human brain mapping 8(4):194-208.

Langan J, Peltier SJ, Bo J, Fling BW, Welsh RC, Seidler RD (2010) Functional implications of age differences in motor system connectivity. Frontiers in systems neuroscience 4:17.

Liu L, Rosjat N, Popovych S, Wang BA, Yeldesbay A, Tóth TI, Viswanathan S, Grefkes CB, Fink GR, Daun S (2017) Age-related changes in oscillatory power affect motor action. PLoS ONE 12(11): e0187911

Makeig S, Bell AJ, Jung TP, Sejnowski TJ (1996) Independent component analysis of electroencephalographic data. Advances in neural information processing systems 145-151.

Mitrushina M, Satz P (1991) Analysis of longitudinal covariance structures in assessment of stability of cognitive functions in elderly. Brain Dysfunction 4:163-173.

Moore RA, Gale A, Morris PH, Forrester D (2006) Theta phase locking across the neocortex reflects cortico-hippocampal recursive communication during goal conflict resolution. International journal of psychophysiology 60(3):260-273.

Nishihara K, Kawai H, Kanemura N, Hara M, Naruse H, Gomi T (2013) A novel approach for evaluating nerve function in healthy elderly persons: A Pilot study 19:309-316.

Nolde SF, Johnson MK, Raye CL (1998) The role of prefrontal cortex during tests of episodic memory. Trends in Cognitive Sciences 2:399-406. 
Nolte G, Bai O, Wheaton L, Mari Z, Vorbach S, Hallett M (2004). Identifying true brain interaction from EEG data using the imaginary part of coherency. Clinical Neurophysiology $115: 2292-2307$.

Oldfield R (1971) The assessment and analysis of handedness: The Edinburgh inventory. Neuropsychologia 9:97-113.

Palva S, Palva JM (2012) Discovering oscillatory interaction networks with M/EEG: challenges and breakthroughs. Trends in cognitive sciences 16(4):219-230.

Pool E-M, Rehme AK, Fink GR, Eickhoff SB, Grefkes C (2013) Network dynamics engaged in the modulation of motor behavior in healthy subjects. NeuroImage 82:68-76.

Popovych S, Rosjat N, Tóth TI, Wang BA, Liu L, Abdollahi RO, Viswanathan S, Grefkes C, Fink GR, Daun S (2016) Movement-related phase locking in the delta-theta frequency band. NeuroImage 139:439-449.

Reuter-Lorenz PA, Cappell KA (2008) Neurocognitive Aging and the Compensation Hypothesis. Current Directions in Psychological Science 17(3):177-182.

Reuter-Lorenz PA, Jonides J, Smith ES, Hartley A, Miller A, Marshuetz C, Koeppe RA (2000) Age differences in the frontal lateralization of verbal and spatial working memory revealed by PET. Journal of Cognitive Neuroscience 12:174-187. 
Rigoni D, Brass M, Roger C, Vidal F, Sartori G (2013) Top-down modulation of brain activity underlying intentional action and its relationship with awareness of intention: An ERP/Laplacian analysis. Exp Brain Res 229:347-357.

Rowe JB, Siebner H, Filipovic SR, Cordivari C, Gerschlager W, Rothwell J, Frackowiak R (2006) Aging is associated with contrasting changes in local and distant cortical connectivity in the human motor system. Neuroimage 32(2):747-760.

Sailer A, Dichgans J, Gerloff C (2000) The influence of normal aging on the cortical processing of a simple motor task. Neurology 55(7):979-985.

Schmorrow DD, Fidopiastis CM (Eds.). (2011). Foundations of Augmented Cognition. Directing the Future of Adaptive Systems: 6th International Conference, FAC 2011, Held as Part of HCI International 2011, Orlando, FL, USA, July 9-14, Proceedings (Vol. 6780). Springer Science \& Business Media.

Seidler RD, Bernard JA, Burutolu TB, Fling BW, Gordon MT, Gwin JT, Kwak Y, Lipps BB (2010) Motor control and aging: links to age-related brain structural, functional and biochemical effects. Neurosci Biobehav Rev 34(5):721-733.

Shibasaki H, Hallett M (2006) What is the Bereitschaftspotential? Clinical neurophysiology 117(11):2341-2356.

Singer W (1999) Neuronal synchrony: A versatile code for the definition of relations? Neuron 24: 49-65. 
Singer W (2004) Synchrony, oscillations and relational codes. The Visual Neurosciences. The MIT Press 1665-1681

Spreen O, Strauss E (1998) A Compendium of Neuropsychological Tests. Administration, Norms, and Commentary. New York:Oxford University Press.

Steinmetz H, Furst G, Meyer BU (1989) Craniocerebral topography within the international 10-20 system. Electroencephalogr Clin Neurophysiol 72:499-506.

Tenke CE, Kayser J, Manna CG, Fekri S, Kroppmann CJ, Schaller JD, Alschuler DM, Stewart JW, Mcgrath PJ, Bruder GE (2012) Current source density measures of EEG alpha predict antidepressant treatment response. Biol Psychiatry 70:388-394.

Uhlhaas PJ, Roux F, Rodriguez E, Rotarska-Jagiela A, Singer W (2010) Neural synchrony and the development of cortical networks. Trends in cognitive sciences 14(2):72-80.

Van Wijk BCM, Beek PJ, Daffertshofer A. Neural synchrony within the motor system: what have we learned so far? Frontiers in Human Neuroscience. 2012;6:252. doi:10.3389/fnhum.2012.00252.

Vinck M, Oostenveld R, van Wingerden M, Battaglia F, Pennartz CMA (2011) An improved index of phase-synchronization for electrophysiological data in the presence of volumeconduction, noise and sample-size bias. Neuroimage 55:1548-1565. 
Wang BA, Viswanathan S, Abdollahi RO, Rosjat N, Popovych S, Daun S, Grefkes C, Fink GR (2017) Frequency-specific modulation of connectivity in the ipsilateral sensorimotor cortex by different forms of movement initiation. NeuroImage, 159, 248-260.

Ward NS, Swayne OB, Newton JM (2008) Age-dependent changes in the neural correlates of force modulation: an fMRI study. Neurobiology of aging 29(9):1434-1446.

Wendel K, Väisänen O, Malmivuo J, et al. (2009) EEG/MEG Source Imaging: Methods, Challenges, and Open Issues. Computational Intelligence and Neuroscience, doi:10.1155/2009/656092

Wu T, Hallett M (2005) The influence of normal human ageing on automatic movements. J Physiol 562(2):605-615. 


\section{Supplementary figure}
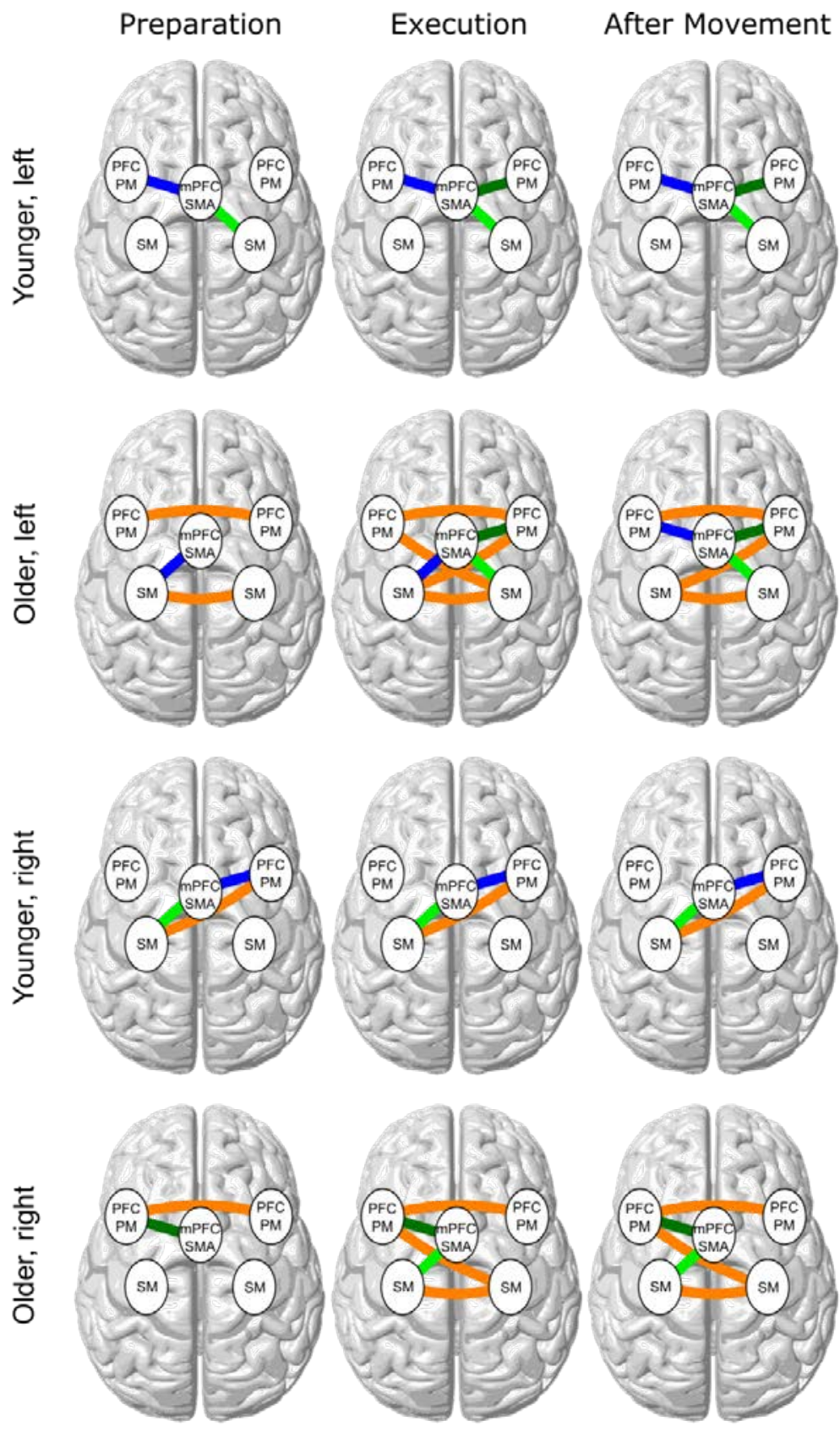

Fig. S1 Summarized phase locking networks for left and right handed tapping in younger and older subjects. Summary of the results presented as a network of five virtual electrodes: left (top two rows) for younger (first row) and older subjects (second row); right (bottom two rows) for younger (third row) and older subjects (fourth row). The networks in each row appeared during the preparation $[-300,0] \mathrm{ms}$, the execution phase of the movement $[0,200] \mathrm{ms}$, and in the post-movement phase [200,500] ms. Connections are labeled as follows: ipsilateral (blue), contralateral SM-mPFC/SMA (light green), contralateral PFC/PM-mPFC/SMA (dark green), and inter-hemispheric (orange). 